\title{
Chemical Modification of Marine Polysaccharide (Alginate) By Free-Radical Graft Copolymerization - a Short Review
}

\author{
A Salisu*1; Ahmedy A. Naim ${ }^{1}$; M.M. Sanagi ${ }^{21}$ \\ ${ }^{I}$ Department of Chemistry, Faculty of science, Universiti Teknologi Malaysia, 81310 Johor Bahru, Johor, \\ ${ }^{2}$ Ibnu Sina Institute for Fundamental Science Studies, Nanotechnology Research Alliance, Universiti Teknology \\ Malaysia, 81310 Johor Bahru, Johor,
}

\begin{abstract}
Because of considerable interest in the preparation of biodegradable materials based on natural polymers, many techniques have been used to modify the physical and chemical properties of natural polymers. Due to the presence of various functional groups on molecular chains, polysaccharides could be readily modified chemically to form polymer hybrid of good composition and functional properties. There have been a number of studies reported for chemical attachments of synthetics polymers. However, grafting of vinyl monomers onto polysaccharides backbone via radical polymerization is often used technique. Alginic acid or alginate is polyuronides, i.e., polysaccharides in which the molecules are built up of uronic acid residues which are obtained from seaweed species of brown algae. Alginate has broad applications in various fields such as pharmaceutical, biomedical, agriculture, and environmental. The present review compiled the studies on the chemical modification of alginate via free-radical graft copolymerization, particularly by generating the free radicals using thermal initiator or electron transfer reaction (redox system) and the potential applications of the grafted copolymers.
\end{abstract}

Keywords: alginate, biodegradable, graft copolymerization, polysaccharide, radicals

\section{Introduction}

In recent years there has been an increase interest in biodegradable materials. Biodegradable materials are used in packaging, medicine, agriculture and other applications. Natural polymers such as polysaccharides are available in large quantities from renewable sources. Polysaccharides are carbohydrates that contain many monosaccharide units joined together by glycosidic bonds. The majority of carbohydrates that occur in nature are in the form of polysaccharides. The important roles played by polysaccharides in living organisms are either storage or structural functions. Due to the presence of various functional groups on molecular chains, polysaccharides could be readily modified chemically to form polymer hybrid of good composition and functional properties. The recent investigation of polysaccharide-based materials can be attributed to new synthetic approaches for their modification with the aim to improve their properties for specific applications. These synthetic approaches include the combination of polysaccharides with other polymers. Grafting of vinyl monomers onto polysaccharides via radical polymerization has attracted the interest of many researchers in the last two decades [1]. However, the methods employed for grafting vinyl monomers onto polysaccharides are different from that of grafting non vinyl monomers. Non vinyl graft copolymerization can be achieved through polycondensation reactions; although competitive degradation reaction may severely result to rapid loss of molecular weight in short period of time due to susceptibility of many functional groups of polysaccharides towards acids, bases, and reducing agents or in most cases low yield of product is obtained. Most of the recent researches on the chemical modification of polysaccharides involve the introduction of synthetic polymer on the backbone through grafting techniques. The radical-initiated polymerization has gained considerable attention, and has proven to be useful methods for the industrial production of modified polysaccharides. Recently, many review papers have been published on the modification of polysaccharides. However, this article is intended to bring out the researches on the chemical modification of marine polysaccharide (i.e. alginate) by free- radical graft copolymerization particularly on "grafting from" method with major emphasis on radical formation by thermal or electron transfer reaction (redox system) and their potential application.

\subsection{Synthesis And Characterization Of Graft Copolymers}

Graft copolymer is a macromolecular chain in which one or more blocks are connected to the main chain (called trunk polymer) as side chain(s). Thus, it can be described as having the general structure illustrated schematically in Fig 1., where the main polymer backbone poly(A), commonly referred to as the trunk polymer, has branches of polymer chain $\operatorname{poly}(\mathrm{B})$ originating from different points along its length. The simple way to describe this structure is poly(A)-graft-poly(B), which can be further condensed as poly(A)-g$\operatorname{poly}(\mathrm{B})$. 


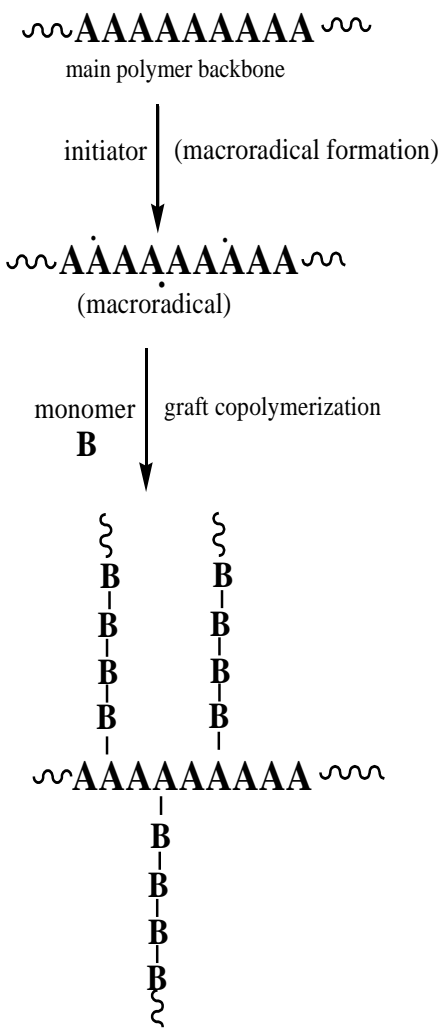

Poly(A)-g-Poly(B)
Fig. 1 General free radical mechanism of graft copolymerization of vinyl monomer $B$ onto main polymer backbone Poly(A) to form the graft copolymer Poly(A)-g-poly(B).

Polymer chains are grafted on polysaccharides through three main methods: the "grafting through", the "grafting onto" and the "grafting from" processes. The grafting through technique involves copolymerizing premade vinyl functionalized polysaccharide material with comonomers. The

"grafting onto" technique requires the presynthesis of end-functionalized linear chains that are subsequently covalently bonded to the polysaccharide. However, this method usually suffers from low grafting density (due to steric hindrance). The "grafting from" involving the growth of grafts directly from the polysaccharide backbones is the most extensively studied and used technique [2-5].

The separation of the copolymer is very essential before characterization of degree of grafting and requires selection of a suitable organic solvent and involves prolonged soxhlet extraction to remove the homopolymer from the surface of the copolymer.

The grafting is generally evaluated as the increase in the weight of the polysaccharide on grafting vinyl monomer onto the backbone. Although, there are many ways of expressing this increase, but the most important include; the percentage of grafting, $\mathrm{G}(\%)$, and yield of graft copolymerization, Y (\%) which can be calculated using equations (I) and (II) respectively [6-7].

$$
\begin{aligned}
& \mathrm{G}(\%)=\frac{\mathrm{W}_{2}-\mathrm{W}_{1}}{\mathrm{~W}_{1}} \times 100 \ldots \ldots . \ldots(\mathrm{I})
\end{aligned}
$$

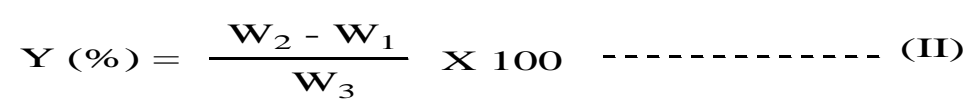

Where $\mathrm{W}_{1}$ is the original weight of the substrate, $\mathrm{W}_{2}$ is the weight of the product after soxhlet extraction and $\mathrm{W}_{3}$ is the weight of vinyl monomer.

1.2 Alginic Acid:Alginic acid also called alginate is among the most abundant natural polysaccharides in nature. Usually alginic acid is obtained from various seaweeds species of brown algae [8]. Although, some bacteria mostly the representatives of the genera Azobacter and Pseudomonas also produce alginic acids [9]. Alginic acid is a linear copolymer consisting mainly the residue of $\beta$-1,4-linked-D-mannuronic acid (M-Block) and $\alpha$-1,4-linked-L-guluronic acid (G-Block) arranged in an irregular blockwise pattern of varying proportions of GG, MG, and MM blocks as represented in fig. 2 [10]. The copolymer composition, sequence and molecular weight vary with species and growth conditions. Because of the abundance of algae in water bodies, large amount of alginate material exist in nature. Their content in the biomass may amount to $40 \%$ of the dry weight. Industrial alginate production is approximately 50,000 metric tons annually and almost $30 \%$ of this amount is devoted to the food industry, the remaining being used in indusrial, pharmaceutical, biotechnology and dental applications [11]. By virtue of their unique physicochemical properties, alginic acids and their salts have found wide range of application in pharmaceutical [12-13], biomedical [14-15], agriculture [16] and purification [17- 
19] Therefore, in recent years, there is considerable interest towards the chemical modification of alginate to enhance its functional properties. Alginate has a number of free hydroxyl and carboxyl groups distributed along the polymer backbone, therefore it could be readily derivatized to form polymer hybrid of good architecture, composition and functional properties. Chemical modification of alginate has been achieved through various methods especially by polycondensation reactions such as esterification, amidation, amination as well as by grafting by radical polymerization. Alginate is extremely important biopolymer that attracted the interest of many researchers in the field of pharmaceutical, biotechnology and bioengineering

$\mathbf{a}$

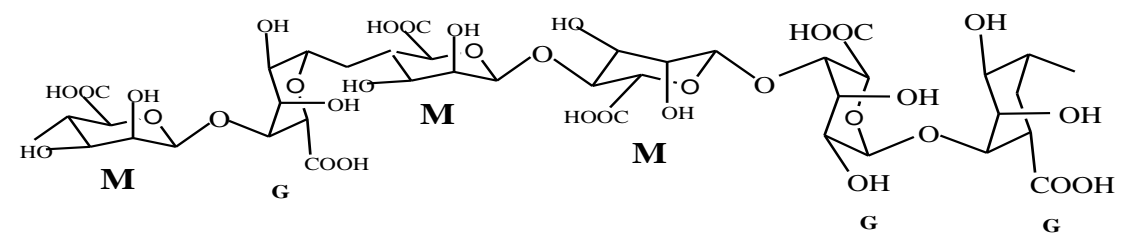

b

$\underbrace{M M M M G M G G G G G M G M G G G G G G G G M M G M G M G M ~}$

G-block

G-block

MG-block

Fig. 2. Representative structure alginic acid: (a) chain conformation and (b) block distribution

1.2.1 Physical Properties Of Alginic Acids:Alginic acids form water-soluble salts with monovalent cations but are precipitated upon acidification. Alginate of many divalent cations notably of $\mathrm{Ca}^{2+}, \mathrm{Sr}^{2+}$ and $\mathrm{Ba}^{2+}$ are insoluble in water. This properties is utilized in the isolation of alginic acid from algae [20]. The solubility of alginic acid in water is dictated by three parameters, first $\mathrm{pH}$ of the solvent, next is ionic strength of the medium and presence of gelling ions in the solvent. In order to make alginic acid soluble in water the $\mathrm{pH}$ should be above a certain critical value which enable the carboxylic groups be deprotonated. It is worthy of note that changing the ionic strength of the medium greatly affects the solubility of alginic acids; because alginate is known to form a physical gel by hydrogen bonding at low $\mathrm{pH}$ (acid gel), and also chelates with divalent cations which act as crosslinkers between adjacent polymer chains. G- block residues are the ones mainly responsible for such ionic interactions, in addition to G- blocks, MG blocks also participate in forming weak junctions [21]. The affinity of alginate towards divalent cations decreases in the following order: $\mathrm{Pb}>\mathrm{Cu}>\mathrm{Cd}>\mathrm{Ba}>\mathrm{Sr}>\mathrm{Ca}$ $>\mathrm{Co}>\mathrm{Ni}, \mathrm{Zn}>\mathrm{Mn}$ [19]. However, $\mathrm{Ca}^{2+}$ ion is the most commonly used cation to induced alginate gel formation.

However, recently there has been a report on chemical modification of alginate in organic solvent systems. It has been observed that the tetrabutylammonium salt of alginic acid (TBA-alginate) is only partially soluble in Dimethylsulfoxide (DMSO). However, in the presence of a dissolution promoter such as Tetrabutylammoniumflouride (TBAF), the TBA salts of alginic acid were found to be completely soluble in the following organic solvents: Dimethylsulfoxide (DMSO), Dimethylfomamide (DMF), and Dimethyl-2imidazolidinone (DMI) [22].

1.3 Graft Copolymerization Of Alginic Acid:Alginic acid modification is usually carried out at the two secondary C-2 and C-3 hydroxyl groups as well as C-6 carboxylic acid group. Chemical modification of polysaccharides by radical polymerization involves generation of radicals on the backbone (trunk polymer) in the presence of initiator, and then allowing these radicals to serve as macro initiators for the polymerization of vinyl monomers [23]. Shi et al. [24] reported the synthesis of superabsorbent composite material by graft copolymerization of aqueous solution of partially neutralized acrylic acid and styrene onto sodium alginate backbones using ammonium per sulfate (APS) as the initiator and methylenebiscrylamide (MBA) as the crosslinker in the presence of attapulgite. Investigation on the effect of salts on the swelling behaviors shows that the swelling in $\mathrm{NaCl}$ solution was controlled by osmotic pressure, whereas in $\mathrm{CaCl}_{2}$ and $\mathrm{AlCl}_{3}$ solution, the composite undergoes swelling-deswelling process which was indused by stronger ionic interaction between carboxylate ion and $\mathrm{Ca}^{2+} / \mathrm{Al}^{3+}$ ion. The swelling ratio of the composite also shows good buffer action in an extended $\mathrm{pH}$ value range from 4 to 11 and exhibited smart responsive characteristics in $0.1 \mathrm{M}$ buffer solution with $\mathrm{pH} 2.2$ and 7.4 and these properties could be utilized for drug delivery carrier. Yadav et al. [25] reported chemical modification of alginate through grafting of 2-acrylamidoglycolic acid (AGA) by radical polymerization using potassium peroxydiphosphate (PDP) and silver nitrate $\left(\mathrm{AgNO}_{3}\right)$ as redox system. They achieved high grafting ratio of $415 \%$ under optimized conditions (alginate $1.0 \mathrm{~g} / \mathrm{dm}^{3}$, PDP $0.016 \mathrm{M}$, AGA 0.0.073M, Ag+ 0.0044M, at $40^{\circ} \mathrm{C}$ for $3 \mathrm{hr}$ ). Graft copolymers formed show better results for swelling and flocculating properties in comparison to alginic acid. Swelling behavior of the graft copolymers were found to be dependent on the monomer concentration which results in the attachment of longer pendant chains of 2- 
acrylamidoglycolic acid, that is responsible for maximum hydrophilic character in graft copolymer thereby increasing the value of percentage swelling and swelling ratio. They determined metal ion sorption behavior in terms of percentage ion uptake, partition coefficient, and retention capacity. Metal ion sorption character was found to be higher as percentage of grafting increases. However, the results show that $\mathrm{Pb}^{+2}$ was least absorbed in comparison to four metal ions studied. Sand et al. [26] also reported the synthesis of alginate- grafted vinylsulfonic acid (VSA) by radical polymerization using potassium peroxydiphosphate (PDP) and thiourea (TU) redox system. The optimum conditions were (PDP $0.010 \mathrm{M}, \mathrm{TU} 0.0048 \mathrm{M}$, alginate $1.6 \mathrm{M}$, VSA $0.046 \mathrm{M}$ at $50^{\circ} \mathrm{C}$ for $2 \mathrm{hr}$ ). The copolymer formed shows better results for swelling, metal ion uptake, flocculating and resistance to biodegradability in comparison to alginate. Pourjavadi et al. [27] prepared a novel superabsorbent composite based on sodium alginate and the inorganic clay kaolin via the graft copolymerization of acrylic acid in an aqueous medium with methylene biscrylamide (MBA) as a crosslinking agent and ammonium persulfate (APS) as an initiator. The results indicate that the superabsorbent hydrogel has relatively good potential to hold water and saline solutions. Mishra et al. [18] reported modification of alginate by grafting N-vinyl-2-pyrrolidone using potassium peroxymonosulphate/glycolic redox initiator. Maximum grafting has been obtained at $\left(0.1 \mathrm{~g} / \mathrm{dm}^{3}\right.$ concentration of alginate, $0.16 \mathrm{M}$ concentration of $\mathrm{N}$-vinylpyrrolidone, at $50^{\circ} \mathrm{C}$ for $\left.3 \mathrm{hr}\right)$. The graft copolymer showed enhances metal uptake and flocculation property than pure alginate. Sadeghi et al. [28] also reported the synthesis of biocopolymer based on sodium alginate and polymethacrylonitrile using ceric ammonium nitrate (CAN) initiator in aqueous solution. Optimum conditions were found to be $(0.66 \% \mathrm{w} / \mathrm{v}$ sodium alginate, $0.0004 \mathrm{M} \mathrm{CAN}, 0.9 \mathrm{M} \mathrm{MAN}$, at $60^{\circ} \mathrm{C}$ for $2 \mathrm{hr}$.) under optimized conditions the grafting parameters were $376 \%$. Nuran et al. [29] reported graft copolymerization of itaconic acid (IA) onto sodium alginate using benzoyl peroxide (BPO) as the initiator. The optimum grafting conditions for maximum yield were obtained with reaction time of $1 \mathrm{hr}$, reaction temperature of $85^{\circ} \mathrm{C}$, IA concentration of $1.38 \mathrm{M}$, BPO concentration of $0.0182 \mathrm{M}$ and $1.5 \mathrm{~g}$ of sodium alginate. Lan et al. [16] reported a novel gelling method for stabilization of phase change material $\mathrm{Na}_{2} \mathrm{HPO}_{4} \cdot 12 \mathrm{H}_{2} \mathrm{O}$ with sodium alginate grafted sodium acrylate using potassium persulfate/sodiumsulfite redox system. The optimum synthesis conditions to obtained product with stable heat storage performance were $\left(2.8 \% \mathrm{w} / \mathrm{w}\right.$ sodium acrylate, $0.4 \%$ sodium alginate, $0.06 \% \mathrm{PPS}$ at $50^{\circ} \mathrm{C}$ for $2 \mathrm{hr}$ ). Graft copolymerization of alginate with ethylacrylate (EA) using ceric ammonium nitrate as an initiator was reported, the grafting parameters were optimized, the kinetic scheme of free radical graft copolymerization has been proposed and the equations relating the values of rate of polymerization, rate of graft copolymerization and rate of homopolymerization were also suggested. The optimum conditions were (1g sodium alginate, CAN $0.15 \mathrm{M}$, EA $0.4 \mathrm{M}$ at $50^{\circ} \mathrm{C}$ for $4 \mathrm{hr}$ ) [30]. Shah et al. [31] reported graft copolymerization of methyl acrylate (MA) and methyl methacrylate (MMA) onto sodium alginate (SA) with ceric ammonium nitrate (CAN) as a redox initiator in an aqueous medium. The optimized conditions affording the maximum percentage of grafting of methyl acrylate and methyl methacrylate onto sodium alginate were (CAN 0.1M, SA 0.1g, MA 0.453M, MMA $0.407 \mathrm{M}$ at $50^{\circ} \mathrm{C}$ for $4 \mathrm{hr}$ ). A comparison of the reactivity of the acrylates monomers with that of acrylonitrile has been made. Shah et al. [32] also reported graft copolymerization of acrylonitrile onto sodium alginate in aqueous medium using ferrous ammonium-hydrogen peroxide (Fenton's reagent) as the redox initiator. The concentration of sulfuric acid, initiator, monomer, temperature and time were optimized. A pH- sensitive graftcopolymer of polyacrylamide and sodium alginate was synthesized by free radical polymerization using ammonium persulfate (APS) initiator under nitrogen atmosphere followed by alkaline hydrolysis. The synthetic pathway of polyacrylamide-g-sodium alginate copolymer is represented in Fig.3. The study indicated that the copolymer exhibits considerable $\mathrm{pH}$-sensitive behavior, this property could be utilized in drug delivery system [33].

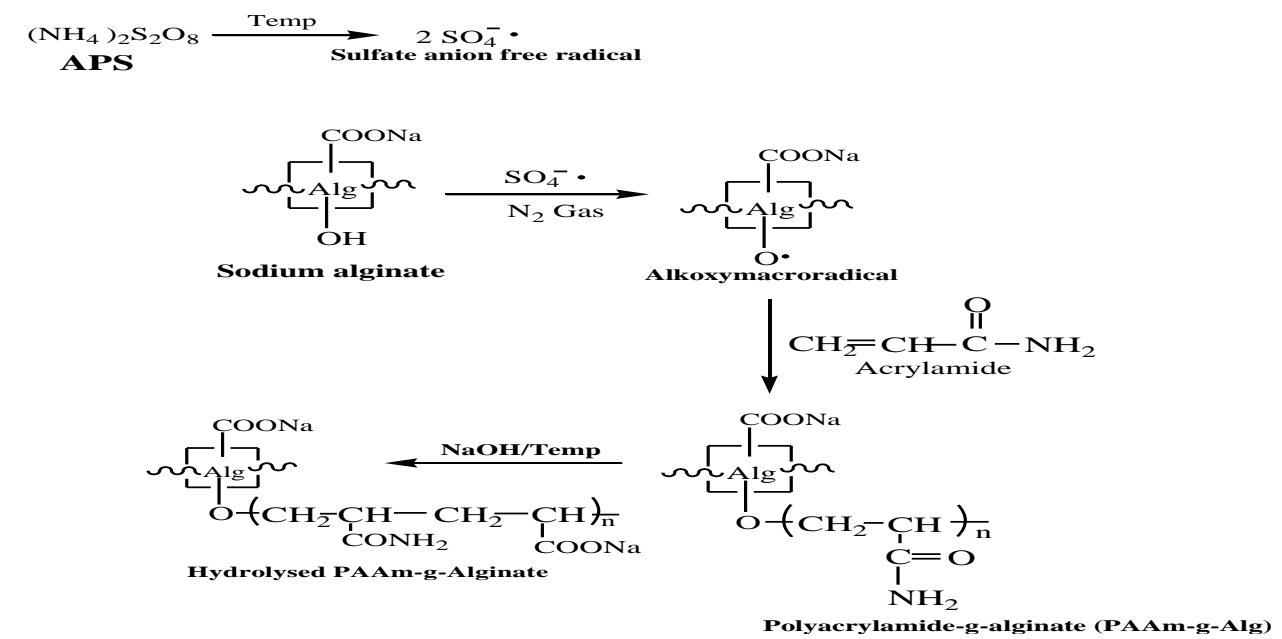

Fig.3. Proposed mechanistic pathway for the synthesis of $\mathrm{pH}$ - sensitive PAAm-g-alginate copolymer 


\section{Conclusion}

Alginate is one of the most abundant natural polysaccharides of marine origin. The graft copolymers of this polysaccharide find applications in diverse fields including pharmaceutical, biomedical, agriculture, and environmental. The possibility of modification of alginate provides greater opportunity for obtaining hybrid materials for specific applications. Polymers with promising applications in the biomedical field as delivery systems of therapeutic agents, bioseparation devices have been attracting much attention, due to non-toxic nature of alginate. Furthermore, biodegradable polymers have been attracting much attention in the present time, due to increase concern on environmental pollution created by plastic waste. Therefore, grafting synthetic polymers onto the backbone natural polymers will lead to the degradation of synthetic plastics to due microbial action.

This article presented the compilation of the most recent published papers on chemical modification of marine polysaccharide (alginate) via radical graft copolymerization method. In recent years, many researchers paid much attention to the graft copolymerization of natural polysaccharides in order to synthesize novel tailored hybrid materials. Although, there are many ways of achieving this goal, but chemical modification by radical polymerization prove to be the method of choice to many researchers due its facile reaction conditions. Graft copolymerization of vinyl monomers onto to polysaccharides are found to be greatly influenced by initiator concentration, monomer concentration, reaction temperature and time. Despite the problem encountered with this method which results in ill-defined materials and homopolymer formation, however, this could be minimized by using reversible-deactivation radical polymerization (RDRP) that allow the preparation of welldefined materials. This contribution is made in order to stimulate further research on chemical modification of polysaccharides particularly by employing radical polymerization method.

\section{References}

[1]. A Sand, M. Yadav, D.K. Mishra, and K. Behari, Alginic acid-g-poly(N-vinylformamide) graft copolymer: Synthesis, characerization, swelling, and flocculation property. Journal of Applied Polymer Science, 121(3), 2011, 1400-1407.

[2]. M. Tizzotti, A. Charlot, E. Fleury, M. Stenzel, and J. Bernard, Modification of Polysaccharides Through Controlled/Living Radical Polymerization Grafting - Towards the Generation of High Performance Hybrids. Macromolecular Rapid Communications, 31(20), 2010 1751-1772.

[3]. V. Athawale, and S. Rathi, Graft polymerization: starch as amodel substrata JMS-Rev. macro-mol Chem. Phys., C39, 1999, 44580 .

[4]. D. Jenkins, andS. Hudson, Review of vinyl graft copolymerization featuring recent advances toward controoled radical-based reactions and illustrated with chitin/chitosan trunk polymers. Chem. Rev., 101: 2001, 3245-73.

[5]. A. Berlin, and V. Kislenko, Kinetics and mechanism of radical graft polymerization of monomers onto polysaccharides. progress in Polymer Science, 17: 1992, 765-825.

[6]. J.M. Fang, P.A. Fowler and C.A.S. Hill, Studies on the grafting of acryloylated potato starch with styrene. Journal of Appl. Polym. Sci., 96, 2005, 452-459.

[7]. V. Singh, A. Tiwari, S. Pandey, and S.K. Singh, Microwave accelerated synthesis and characterization of potato starch-gpoly(acrylamide). Starch/Starke, 58, 2006 536-543.

[8]. H. Ertesvåg and S. Valla, Biosynthesis and applications of alginates. Polymer Degradation and Stability, 59(1-3), 1998, 85-91.

[9]. A. Linker and R. Jones, Nature, 204, 1964, 187.

[10]. T. Matsumoto, M. Kawai, and T. Masuda, influence of concentration and mannuronate/guluronate [correction of gluronate] ratio on steady flow properties of alginate aqueous systems. Biorheology, 29: 1992, 411-417.

[11]. K. Draget, Alginate In : Hand book of hydrocolloids. 2009, 375-95.

[12]. J.P. Chen, I.M. Chu, M.Y. Shiao B.R.S. Hsu, and S.H. Fu, Microencapsulation of islets in PEG-amine modified alginate-poly(1lysine)-alginate microcapsules for constructing bioartificial pancreas. Journal of Fermentation and Bioengineering, 86(2), 1998, $185-190$.

[13]. J.H. Kim, S.B. Lee, S.J Kim, and Y.M . Lee, Rapid temperature/pH response of porous alginate-g-poly(N-isopropylacrylamide) hydrogels. Polymer, 43(26), 2002, 7549-7558.

[14]. A. Bhattacharya and B.N. Misra Grafting: a versatile means to modify polymers: Techniques, factors and applications. Progress in Polymer Science, 29(8),2004, 767-814.

[15]. K.Y. Lee and D.J. Mooney, Alginate: Properties and biomedical applications. Progress in Polymer Science, 37(1), 2012, $106-126$.

[16]. X.Z. Lan, Z.C Tan, Q. Shi, and C.G. Yang, A novel gelling method for stabilization of phase change material $\mathrm{Na}_{2} \mathrm{HPO} \cdot 12 \mathrm{H}_{2} \mathrm{O}$ with sodium alginate grafted sodium acrylate. Thermochimica Acta, 2007. 463(1-2), 2007, 18-20.

[17]. A.J.M. Al-Karawi, Z.H.J. Al-Qaisi, H.I. Abdullah, A.M.A. Al-Mokaram and D.T.A. Al-Heetimi, Synthesis, characterization of acrylamide grafted chitosan and its use in removal of copper(II) ions from water. Carbohydrate Polymers, 83(2), 2011, 495-500.

[18]. D.K. Mishra, S. Arpit, Y. Mithilesh, and B. Kunj, Modification of Alginate by grafting of N-vinyl-2-pyrrolidone and studies of physicochemical properties in terms of swelling capacity, metal-ion uptake and flocculation. Carbohydrate Polymers, 80, 2010, 1147-1154.

[19]. Y. Morsh, T. Donati, B. Strand, and G. Skjak-Braek, Effect of $\mathrm{ca}^{2+}, \mathrm{Ba}^{2+}, \mathrm{Sr}^{2+}$ on alginate microbeads. Biomacromolecules, 7, 2006, $1471-80$.

[20]. V. Chapman and D. Chapman, Seaweeds and their uses. London: Chapman and Hall 1980.

[21]. T. Donati, S. Holtans, Y. Morsh, M. Bergogna and M.S.B Dentini, New hypothesis on the role of alternating sequences in calcium alginate gels. Biomacromolecules, 16, 2005, 1031-40.

[22]. N.P. Siddhesh, and J.E. Kevin, Chemical Modification of Alginates in Organic system. Biomacromolecules 12, 2011, 4095-4103.

[23]. M. Sabyasachi, R. Somdipta and S. Biswanath, Polysaccharide-Based Graft Copolymers in Controlled Drug Delivery. International Journal of phar.Tech Research, 2, 2010, 1350-1358.

[24]. X. Shi, W. Wang, Y. Kang, and A. Wang, Enhanced swelling properties of a novel sodium alginate-based superabsorbent composites: NaAlg-g-poly(NaA-co-St)/APT. Journal of Applied Polymer Science, 125(3), 2012, 1822-1832. 
[25]. M. Yadav, D.K. Mishra, A. Sand, and K. Behari, Modification of alginate through the grafting of 2-acrylamidoglycolic acid and study of physicochemical properties in terms of swelling capacity, metal ion sorption, flocculation and biodegradability. Carbohydrate Polymers, 84(1), 2011, 83-89.

[26]. A. Sand, M. Yadav and K. Behari, Synthesis and characterization of alginate-g-vinyl sulfonic acid with a potassium peroxydiphosphate/thiourea system. Journal of Applied Polymer Science, 118(6), 2010, 3685-3694.

[27]. A. Pourjavadi, H. Ghasemzadeh and R. Soleyman, Synthesis, characterization, and swelling behavior of alginate-g-poly(sodium acrylate)/kaolin superabsorbent hydrogel composites. Journal of Applied Polymer Science, 105(5), 2007, 2631-2639.

[28]. M. Sadeghi, G. Nahid and S. Fatemeh, Optimization of Synthetic Conditions of a Novel Graft Copolymer Based on Alginate. European Journal of Scientific Research, 64, 2011, 587-597.

[29]. I. Nuran, K. Fatma and I. Murat, Graft copolymerization of itaconic acid onto sodium alginate usin benzoyl peroxide. Carbohydrate Polymers, 79, 2010, 665-672.

[30]. S.B. Shah, P.P Chandrakant and H.C. Trivedi, Ceric-induced grafting of ethyl-acrylate onto sodium alginate. Die Angewandte Makromolekulare Chemie, 214, 1994, 75-89.

[31]. S.B. Shah, C.P. Patel, and H.C. Trivedi, Ceric-induced grafting of acrylate monomers onto sodium alginate. Carbohydrate Polymers, 26(1), 1995, 61-67.

[32]. S.B. Shah, C.P. Patel and H.C. Trivedi, Fenton's reagent-initiated graft copolymerization of acrylonitrile onto sodium alginate. Journal of Applied Polymer Science, 1994. 51(8), 1994, 1421-1426.

[33]. V.K. Raghavendra and S. Biswanath, Polyacrylamide-Grafted-Alginate-Based pH- sensitive Hydrogel Beads for Delivery of Ketoprofen to the Intestine. journal of Biomedical Materials Science, 20, 2009, 235-251. 